\title{
Cryptococcal meningitis in a previously healthy child
}

\section{Takondwa Chimowa ${ }^{1}$, Isobel King ${ }^{2}$, Pui-Ying Iroh Tam ${ }^{1,3}$, Carmen Gonzalez-Martinez ${ }^{1,3}$}

1.Department of Paediatrics, Queen Elizabeth Central Hospital, Blantyre, Malawi 2.Mulanje Mission Hospital, Mulanje, Malawi

3.Malawi-Liverpool Wellcome Trust Clinical Research Programme, Blantyre, Malawi

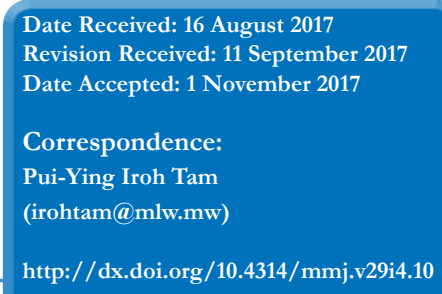

\section{Abstract}

An 8-year-old previously healthy female presented with a 3 weeks history of headache, neck stiffness, deafness, fever and vomiting and was diagnosed with cryptococcal meningitis. She had documented hearing loss and was referred to tertiary-level care after treatment with fluconazole did not improve her neurological signs and symptoms. Her symptoms slowly resolved over two months. This case report illustrates the occurrence of cryptococcal meningitis in a non-immunocompromised patient, as well as the challenges of providing effective care in resource-limited settings.

\section{Case Presentation}

An 8 year-old girl presented with a 3 week history of headache, neck stiffness, deafness, fever and vomiting to Mulanje Mission Hospital. The child was previously well until 3 weeks prior to presentation when she started complaining of headache, which was persistent and kept the child awake at night. There was also associated intermittent fevers and vomiting, especially in the morning. Her mother reported that she had been going to several local clinics with the child, upon the onset of the illness where the child was being treated for malaria, even though the malaria rapid diagnostic tests were negative. As her symptoms were not improving, one local clinic decided to refer the child to the Mission Hospital. At the Mission Hospital, on initial assessment, the child had fever, signs of meningism and deafness. The weight-for-height $z$-score was $\leq 3$ with a middle upper arm circumference (MUAC) of $14.5 \mathrm{~cm}$.

On initial assessment the child was confirmed HIV-negative after three negative antibody rapid tests (Determine ${ }^{\mathrm{TM}}$ and Unigold $\left.^{\text {TM}}\right)$. Subsequent HIV DNA PCR was also done which was negative. Cerebrospinal fluid (CSF) analysis was as follows: WBC $0-3 \times 10^{3} / \mu \mathrm{L}$, acid fast bacilli negative, Gram stain with Gram positive yeast, Indian Ink positive and Cryptococcal antigen positive. The hospital did not have capacity for performing culture. Based on positive Gram stain and Cryptococcal antigen, a diagnosis of cryptococcal meningitis was made, and the child was started on oral fluconazole induction phase at $12 \mathrm{mg} / \mathrm{kg} / \mathrm{day}$. After the initiation of treatment, the child continued to have severe headaches and therapeutic lumbar punctures were done on days 1, 5 and 7. On day 9 there was an improvement on the headaches.

After being on fluconazole for 5 weeks child was transferred to Queen Elizabeth Central Hospital (QECH), a tertiary government referral centre for the Southern Malawi region. The reasons for transfer was firstly, for possible access to first-line fungicidal therapy, and secondly, for work up of possible underlying condition. The family also requested to be referred because they could no longer afford to pay for treatment at the Mission Hospital.

At QECH, on history the child had no recurrent respiratory infections or persistent otitis media, no skin rash or eczema, no prior history of persistent vomiting before this illness, no history of persistent diarrhoea or having bloody stool, and no history of bleeding tendencies. Mother reported that child had normal development and no prior history of poor balance or abnormal gait. On examination the child had a weight of $16 \mathrm{~kg}$, a height of $111.5 \mathrm{~cm}$, with a weight/ height of -2SD. The child had no dysmorphic features, had normal skin (no rash or stigma of previous skin lesions), no telangiectasia, no finger clubbing and had no significant lymphadenopathy. On chest examination, the lung fields were clear and had normal heart sounds with no murmurs. She had a soft and non-tender abdomen with a palpable liver of about $1 \mathrm{~cm}$ below the costal margin with no splenomegaly. Neurologically child was alert, unable to hear properly, and no facial asymmetry. She had normal tone with some tremor in the upper limbs and was noticed to have an ataxic gait.

Tests done at QECH were as follows: FBC: WBC $6.7 \times 10^{3} /$ $\mu \mathrm{L}, \mathrm{RBC} 4.07 \times 10^{3} / \mu \mathrm{L}$, haemoglobin $10.4 \mathrm{~g} / \mathrm{dL}$, haematocrit $32.0 \%$, platelets $330 \times 10^{3} / \mu \mathrm{L}$, total lymphocytes $2.0 \times 10^{3} / \mu \mathrm{L}$ (with total CD 4 count $705 / \mu \mathrm{L}$ with no percentage available), total neutrophils $3.8 \times 10^{3} / \mu \mathrm{L}$, calcium $1.0 \mathrm{mmol} / \mathrm{L}$, random blood sugar $5.4 \mathrm{mmol} / \mathrm{L}$. CSF showed WBC $30 \times 10^{3} / \mu \mathrm{L}$, RBC $3040 \times 10^{3} / \mu \mathrm{L}$, polymorphs $70 \%$, lymphocytes $30 \%$, no organisms were seen on Gram stain, Indian Ink was negative, and there was no growth on culture.

As the child had persistent neurological signs and symptoms as described above, an MRI was done. This showed mild hydrocephalus (Figure 1). Given her clinical improvement, this was managed conservatively. With the presence of hearing deficit, a hearing test was done which showed mild to moderate sensory-neural hearing loss on the left ear.

The child was continued on oral fluconazole $(12 \mathrm{mg} / \mathrm{kg})$ and was discharged after she had stayed in hospital for 17 days. She was seen for follow up in clinic 2 months later, and at that time her hearing impairment was improving and her headache had resolved. Given that no immunodeficiency was identified, the child received induction and maintenance courses of oral fluconazole and completed a total of 15 weeks' treatment.

\section{Discussion}

Cryptococcal meningitis is the most common central nervous system (CNS) fungal infection with high morbidity 


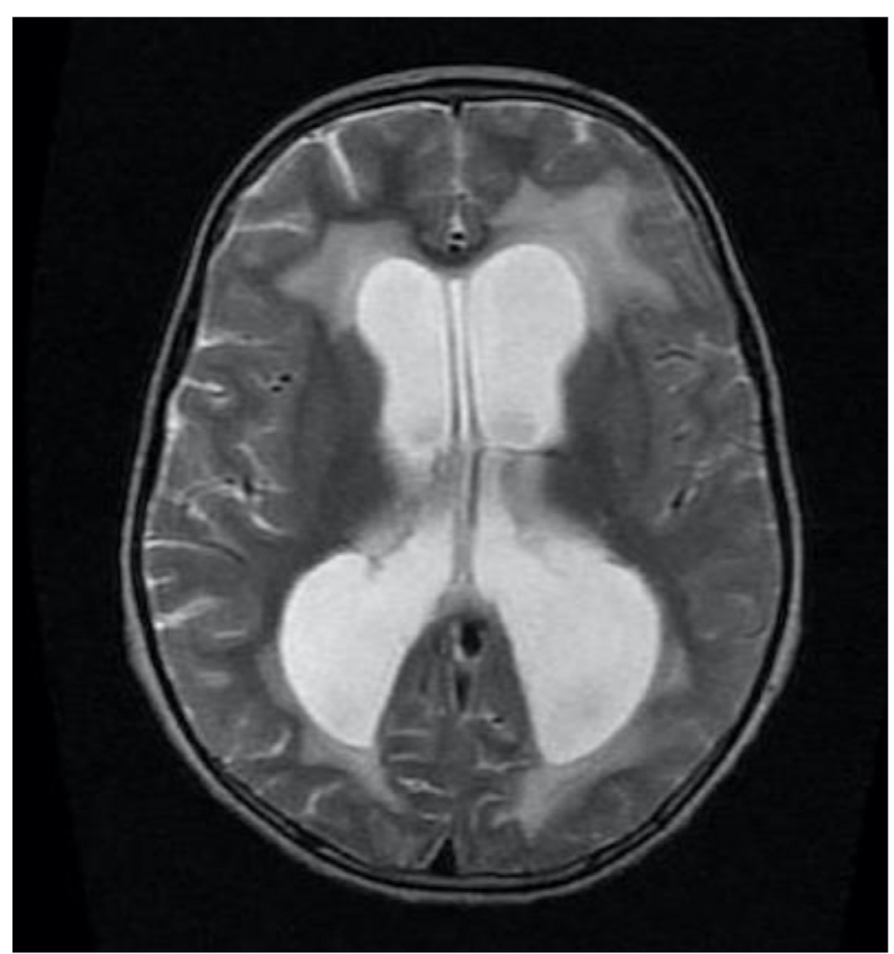

Figure 1. Axial image of MRI brain of patient, illustrating mild hydrocephalus

and mortality. Globally, approximately 957,900 cases of cryptococcal meningitis occur annually, resulting in 624,700 deaths within 3 months of infection in

HIV-infected adults and children ${ }^{1}$. These occur primarily in low-income countries, where health care and resources are limited. The incidence of cryptococcosis in Chinese children is $0.016-100$ cases $/ 100,000$ children $^{2}$. HIV infection is the main risk factor, accounting for $95 \%$ of cases in middleand low-income countries and $80 \%$ of cases in high-income countries. The other risk factors include transplant recipients, patients with acute leukaemias or lymphomas, and those on immunosuppressive therapy including steroids. Cryptococcal infection in immunocompetent host is usually attributed to Cryptococcus gattii while that in immunocompromised is mostly by $C$. neoformans ${ }^{3,4}$. While India ink preparation only has a sensitivity of $86.1 \%$ and specificity of $97.3 \% \%^{5}$, diagnosis of cryptococcal meningitis can be reliably performed by serum cryptococcal latex agglutination titre which has a sensitivity of $97-97.8 \%$ and specificity of $85.9-100 \% 0^{5}$, or by cryptococcal antigen testing in CSF, which has a sensitivity of $100 \%$ and specificity of $97.7 \%$, or serum (sensitivity $100 \%$ and specificity $99.5 \%)^{5}$.

In this case, we did not identify an underlying cause for the cryptococcal meningitis ${ }^{5}$. Diagnosing a primary immunodeficiency in our setting is challenging as laboratory capacity is limited. We followed a pragmatic approach: considering that our patient had reach middle infancy without major problems we focused our differential diagnosis on partial T-cell deficiencies and with the history and simple laboratory tests we evaluated for and confidently excluded the most common conditions (DiGeorge Syndrome, Ataxia Telangiectasia, Wiskott-Aldrich Syndrome and ChediakHigashi Syndrome). We also excluded prevalent secondary causes as HIV infection, leukaemia and diabetes mellitus.

This case stresses the importance of thinking of cryptococcal meningitis in the differential diagnosis of a child who is otherwise immunocompetent presenting with chronic headache, fever and meningeal signs. As we were not able to identify the Cryptococcus species in our patient, there is a possibility $C$. gattii would still be the causative agent, which has been increasingly reported as involved in severe CNS manifestations and classically has been described to affect immunocompetent individuals ${ }^{6}$. In addition to challenges in diagnosis, this case demonstrates the challenges of providing care in resource-limited settings like Malawi where there is limited availability of standard pharmacological agents, and where standard treatment of cryptococcal meningitis is fluconazole monotherapy, which is a fungistatic drug ${ }^{7}$. Best treatment for cryptococcal meningitis in resourcelimited settings is combination therapy with fluconazole and amphotericin $\mathrm{B},{ }^{8}$ a fungicidal drug. However, even at a major referral centre such as ours, there is a lack of effective first-line fungicidal drugs available, and hence only high-dose fluconazole was administered.

In conclusion, diagnosis and treatment of Cryptococcal infection in low resource settings continues to be a challenge and clearly affects the outcome of patients. There is an urgent need to advocate for wide availability of standard and effective fungicidal treatment in low resource settings worldwide so as to reduce morbidity and mortality associated with cryptococcal meningitis.

\section{Authors Contributions}

All authors contributed equally to this work

\section{References}

1.Park BJ, Wannemuehler KA, Marston BJ, Govender N, Pappas PG, Chiller TM. Estimation of the current global burden of cryptococcal meningitis among persons living with HIV/AIDS. AIDS. 2009 Feb 20;23(4):525-30. doi: 10.1097/QAD.0b013e328322ffac.

2.Guo L, Liu L, Liu Y, et al. Characteristics and outcomes of cryptococcal meningitis in HIV seronegative children in Beijing, China , 2002 - 2013. BMC Infect Dis

3.Leal AL, Faganello J, Fuentefria AM, Boldo JT, Bassanesi MC, Vainstein $\mathrm{MH}$. Epidemiological profile of cryptococcal meningitis patients in Rio Grande do Sul, Brazil. Mycopathologia. 2008 Aug;166(2):71-5. doi: 10.1007/s11046-008-9123-2. Epub 2008 Apr 29.

4.Pyrgos V, Seitz AE, Steiner CA, Prevots DR, Williamson PR. Epidemiology of cryptococcal meningitis in the US: 1997-2009. PLoS One. 2013;8(2):e56269. doi: 10.1371/journal.pone.0056269. Epub 2013 Feb 15

5.Nalintya E, Kiggundu R, Meya D. Evolution of Cryptococcal antigen testing: what is new? Curr Fungal Infect Rep 2016;2016:1-6. Epub 2016 Apr 12.

6.Franco-Paredes C, Womack T, Bohlmeyer T, et al. Management of Cryptococcus gattii meningoencephalitis. Lancet Infect Dis. 2015 Mar;15(3):348-55. doi: 10.1016/S1473-3099(14)70945-4. Epub 2014 Nov 26.

7.Dodds Ashley ES, Lewis R, Lwis JS, Martin C, Andes D. Pharmacology of systemic antifungal agents. Clin Infect Dis 2006;43:S28-39.

8.WHO. Rapid advice: Diagnosis, prevention and management of cryptococcal disease in HIV-infected adults, adolescents and children. 2011. http://apps.who.int/iris/ bitstream/10665/44786/1/9789241502979_eng.pdf. Accessed 11 September 2017. 